\title{
RESPUESTA DEL MAÍZ NATIVO DEL ALTIPLANO MEXICANO A PUDRICIÓN DE MAZORCA, BAJO INFECCIÓN NATURAL ${ }^{1}$
}

\author{
Dolores Briones-Reyes ${ }^{2}$,Fernando Castillo-González ${ }^{2}$, José Luis Chávez-Servia ${ }^{3}$ \\ Víctor Héber Aguilar-Rincón ${ }^{2}$, Carlos de León García-de Alba², Antonio Ramírez-Hernández $z^{2}$
}

\begin{abstract}
RESUMEN
Respuesta del maíz nativo del Altiplano mexicano a pudrición de mazorca, bajo infección natural. El objetivo del presente estudio fue evaluar la respuesta a la infección natural por Fusarium spp. en las mazorcas de 55 poblaciones nativas de maíz. Las poblaciones evaluadas procedían de cinco estados de México, estas se sembraron en tres localidades en 2010 y 2011 bajo condiciones de secano (temporal). El porcentaje promedio de pudrición de mazorca fue significativamente diferente entre las poblaciones de maíz, localidades y poblaciones dentro de cada localidad y entre años. Las agrupaciones de poblaciones por color de grano también mostraron diferencias, ya que los grupos de color amarillo y rojo mostraron el menor porcentaje de infección. Por la procedencia geográfica, los maíces de Oaxaca presentaron menor daño que los de los estados de México, Puebla, Tlaxcala y Guerrero. En rendimiento de grano hubo diferencias entre localidades, poblaciones y años. Los efectos significativos en la interacción de grupos de poblaciones por localidad indican que las poblaciones de maíz de origen local tienden a presentar menor porcentaje de pudrición y mayor rendimiento, lo que sugiere un proceso de coevolución y coadaptación. El mayor rendimiento de mazorca se obtuvo en 2011, en donde también destacaron las poblaciones de Oaxaca, por lo que se infiere que las poblaciones nativas de maíz de esta entidad son una fuente potencial de resistencia/ tolerancia a pudrición de mazorca por Fusarium spp.
\end{abstract}

Palabras clave: Zea mays, hospedero-patógeno, diversidad fenotípica, Fusarium spp., hongos fitopatógenos.

\begin{abstract}
Response of native maize from Mexican highlands to ear rot, under natural infection. The objective of this study was to evaluate the response to natural infection of Fusarium spp. on 55 native maize populations. The populations evaluated were collected in five states of Mexico, and planted in three locations in 2010 and 2011 under rainfed conditions. The average percentage of ear rot was significantly different among maize populations, locations and among populations within locations and years. The population groups of kernel color showed differences among them, where yellow and red groups presented the lower infection percentages. In the relation to geographic provenance, maize populations of Oaxaca presented less damage than those from Mexico, Puebla, Tlaxcala and Guerrero states. In grain yield, there were differences among locations, populations and years. In the significant interaction between population groups and locations showed that populations with local origin presented low ear rot percentage and major yield, which suggest a coevolution and coadaptation process. The higher ear weight was obtained in 2011, where the Oaxaca populations were outstanding. It is possible to infer that native maize populations from Oaxaca are a potential source of resistance or tolerance to ear rot caused by Fusarium spp.
\end{abstract}

Keywords: Zea mays, host-pathogen, phenotypic diversity, Fusarium spp., phytopatology fungi.

\footnotetext{
Recibido: 3 de setiembre, 2013. Aceptado: 5 de agosto, 2014. Parte de Tesis de Doctorado de la primer autora, realizada en el Colegio de Postgraduados, México.

2 Colegio de Postgraduados Campus Montecillo. Postgrado de Recursos Genéticos y Productividad-Genética, Postgrado de Fitosanidad-Fitopatología. Carr. México-Texcoco. Km 36.5, Montecillo, Texcoco, Edo. México, CP 56230. briones_2003@yahoo.com.mx, fcastill@colpos.mx, aheber@colpos.mx, cdeleon@colpos.mx, rantonio@colpos.mx

3 Instituto Politécnico Nacional, CIIDIR Unidad Oaxaca, Hornos \# 1003, Santa Cruz Xoxocotlán, Oaxaca, México. C. P. 71230. jchavezs@ ipn.mx
}

(C) 2015 Agronomía Mesoamericana es desarrollada en la Universidad de Costa Rica y se encuentra licenciada con Creative Commons Reconocimiento-NoComercial-SinObraDerivada 3.0 Costa Rica. Para más información escríbanos a pccmca@ucr.ac.cr 


\section{INTRODUCCIÓN}

El cultivo de maíz (Zea mays L.) ocupa el primer lugar por área cultivada en México, principalmente bajo condiciones de secano (temporal) (83\%), la cual presenta rendimientos bajos (2,24 t/ha) determinados por efectos bióticos y abióticos adversos. No obstante, estas áreas aportan 58\% de la producción nacional, la que en el año 2013 ascendió a 22663 953,35 t (SIAP, 2013).

Las poblaciones nativas de maíz, comúnmente denominadas criollas, han sido preservadas por los productores a través de numerosas generaciones. En más del $80 \%$ del área sembrada con maíz en México, prevalecen estas poblaciones, en donde coexisten con organismos benéficos y patógenos en condiciones microecológicas específicas, factores que han permitido la conservación de la diversidad genética del cultivo (Castillo et al., 2000).

Los maíces nativos son importantes porque hacen posible la subsistencia de los pequeños productores en zonas marginadas y marginales, constituyen la base genética de las semillas mejoradas. Además, presentan adaptación a la condición ecológica de su origen, esto como resultado histórico de la evolución bajo domesticación y de la aplicación del conocimiento de los agricultores pocas veces reconocido (Hernández, 1985).

Entre las enfermedades de importancia económica se destaca la pudrición de mazorca causada por Fusarium spp., ya que causa pérdidas de rendimiento de 23 a 30\% (González et al., 2007). Las especies de Fusarium presentan distribución cosmopolita, son endémicas en las regiones maiceras, capaces de colonizar todas las partes de la planta y sobrevivir largos períodos en restos vegetales (Thomas y Buddenhagen, 1980; Desjardins et al., 1994). Las especies de mayor prevalencia son $F$. verticillioides, $F$. graminearum y F. subglutinans las que además de deteriorar el grano, producen micotoxinas que afectan la salud humana y animal (Robledo et al., 2001; Desjardins et al., 2006; Gimeno y Martins, 2011). En México (Gallardo et al., 2006), Irán (Farhang et al., 2007), Vietnam (Trung et al., 2008) y Argentina (Presello et al., 2007), esta enfermedad cobra cada vez mayor importancia. La resistencia genética a la pudrición de mazorca por Fusarium spp. es de tipo cuantitativa y, por lo tanto, altamente influenciada por el ambiente (Nankam y Pataky, 1996; Ali et al., 2005).
La relación de un microorganismo con su hospedero involucra interacciones donde participa el medio ambiente, la enfermedad resulta del ataque de patógenos a hospederos susceptibles, a través de la expresión de factores de virulencia y un reconocimiento por el hospedero que induce una respuesta benéfica o dañina con el objetivo de eliminar el patógeno (Rocha et al., 2004). En las interacciones parásito-hospedero se asume que existe una coevolución y coadaptación como producto de las asociaciones (Palau, 2000).

En diversos países, se han encontrado una gran diversidad de especies de Fusarium infectando mazorcas y granos de maíz (Mesterházy et al., 2012), y también en México se ha documentado la diversidad de especies de Fusarium en poblaciones nativas de maíz (Morales-Rodríguez et al., 2007; García-Aguirre y Martínez-Flores, 2010); este hecho se asume como interacciones coevolutivas de Fusarium y poblaciones de maíz nativo debido a la siembra continua de maíz en las parcelas infestadas. Este tipo de interacciones Fusarium-maíz se ha documentado en otros trabajos (Oren et al., 2003; Desjardins et al., 2007).

Es de suponer que la variación genotípica del maíz, en la que se sustentan las miles de variantes que se encuentran en México, y que han coevolucionado con variantes locales de Fusarium spp., ha dado lugar a un proceso de coadaptación. Este fenómeno hace factible el mejoramiento genético de las poblaciones nativas locales, con base en estrategias participativas de selección, desarrolladas para incrementar la capacidad productiva y reducir problemas fitosanitarios de manera efectiva y durable (Castillo et al., 2000; Smith et al., 2001).

La exploración, estudio y conservación de las poblaciones nativas de maíz son de gran importancia, tanto para conocer los patrones de variación conjunta (patosistema), así como para utilizar la variación genética para resolver el problema de pudrición de mazorca, mediante resistencia sin buscar fuentes genéticas externas, al mismo tiempo que se conserva la diversidad del maíz y se continúa con su evolución dinámica y progresiva (Castillo et al., 2000).

El objetivo del presente estudio fue evaluar la respuesta a la infección natural por Fusarium spp. en las mazorcas de 55 poblaciones nativas de maíz. 


\section{MATERIALES Y MÉTODOS}

\section{Material genético}

Se evaluaron 55 poblaciones de maíz nativo recolectadas con productores en diecinueve localidades ubicadas entre 1498 y 2520 msnm, en cinco Estados de la República Mexicana: trece de la región Montaña de Guerrero, once del sureste del Estado de México, trece de la Mixteca alta de Oaxaca, seis de Puebla y doce de Tlaxcala, y el híbrido azul "San Miguel" como testigo. En México, las coloraciones de grano están asociadas a diferentes formas de preparación de platillos o productos derivados (p. ej. pozole, pinole, téjate, tamales, etc.) (Boege, 2010) y también existen diferencias en aminoácidos y proteínas entre poblaciones con diferente color de grano (VeraGuzmán et al., 2012). En este contexto, se hizo una clasificación de las poblaciones de maíz con base en el color del endospermo/pericarpio, las poblaciones se clasificaron de la siguiente forma: veintidós blancos cristalinos (BC), once azules (A), seis amarillos (Am), cuatro cremas (C), seis blancos anchos harinosos (BA) y seis rojos (R) (Cuadro 1).

\section{Ciclos de cultivo y localidades de evaluación}

Los experimentos se establecieron en los ciclos agrícolas de primavera-verano en los años 2010 y 2011, en condiciones de temporal e infección natural por inóculo local, en tres localidades donde la enfermedad se presenta consistentemente: 1) Campo Experimental del Colegio de Postgraduados, en Montecillo (Mont), Texcoco, Méx. (19 29' N y $98^{\circ}$
54' O, $2250 \mathrm{msnm}$ ) cuyo clima es semiárido, con lluvias en verano, temperatura media anual de 14,6 ${ }^{\circ} \mathrm{C}$ y $558 \mathrm{~mm}$ de precipitación media anual, el suelo es franco arenoso; 2) Ayapango (Aya), Méx., (19 $10^{\prime} \mathrm{N}$ y $98^{\circ} 45^{\prime} \mathrm{O}, 2450 \mathrm{msnm}$ ), donde el clima es subhúmedo con precipitación que va de $\operatorname{los} 800$ a $900 \mathrm{~mm}$ y su temperatura media anual se encuentra entre $\operatorname{los} 12^{\circ}$ y $18{ }^{\circ} \mathrm{C}$; el tipo de suelo predominante es el regosol, dedicado principalmente a la agricultura de temporal y 3) Huamelulpan (Oax), Oaxaca, México ( $17^{\circ} 24^{\prime} \mathrm{N}$ y $97^{\circ} 36^{\prime} \mathrm{O}, 2172 \mathrm{msnm}$ ), es un poblado de zonas boscosas, tiene un clima de templado a frío, con temperaturas mínima y máxima promedio de $10^{\circ}$ y $24^{\circ} \mathrm{C}$, precipitación promedio de $770 \mathrm{~mm}$, el suelo se clasifica en: pedregoso, arcilloso, barroso y la gran mayoría áridos (García, 1973; INAFED, 2014).

\section{Diseño experimental}

Se utilizó un diseño de bloques completos al azar, con dos repeticiones en 2010 y con tres en 2011, en la distribución en campo de las poblaciones de maíz. El bajo número de repeticiones obedeció al limitado tamaño de muestra donado por los agricultores al momento de la colecta. En ambos experimentos la parcela experimental consistió de dos surcos de $5 \mathrm{~m}$ de longitud y $0,90 \mathrm{~m}$ de ancho, con dos plantas cada $0,50 \mathrm{~m}$, distribución que corresponde a la densidad de 45000 plantas/ha. Las semillas fueron desinfestadas superficialmente, mediante inmersión en hipoclorito de sodio al $10 \%$ durante diez minutos, y tratadas con el fungicida Captan (75 g/kg semilla), con el fin de reducir la posibilidad de transportar inóculo.

Cuadro 1. Origen geográfico y color de grano de 55 poblaciones de maíz nativo, colectadas en el Altiplano Mexicano. 2010.

\begin{tabular}{|c|c|c|c|c|c|c|c|c|c|}
\hline \multicolumn{2}{|r|}{ Procedencia geográfica } & \multirow{2}{*}{$\begin{array}{c}\text { Rango de } \\
\text { altitud (msnm) }\end{array}$} & \multicolumn{7}{|c|}{ Color endospermo/pericarpio $*$} \\
\hline Estado & Municipios & & Am & $\mathbf{A}$ & BC & BA & $\mathbf{C}$ & $\mathbf{R}$ & Total \\
\hline Tlaxcala & Ixtacuixtla, Españita, Nanacamilpa, Totolac. & $2220-2720$ & 1 & 3 & 7 & 0 & 1 & 0 & 12 \\
\hline Guerrero & Chilapa, Cualac, Tixtla, Zitlala. & $1498-2222$ & 1 & 3 & 2 & 4 & 0 & 3 & 13 \\
\hline Puebla & Xochitlán y Libres. & $1610-2400$ & 2 & 1 & 3 & 0 & 0 & 0 & 6 \\
\hline Oaxaca & Huamelulpan,Tayata, Peñasco, Ticuá, Tataltepec. & $2144-2290$ & 2 & 3 & 5 & 0 & 0 & 3 & 13 \\
\hline México & Ayapango, Chalco, Tepetlixpa, Texcoco. & $2249-2450$ & 0 & 1 & 5 & 2 & 3 & 0 & 11 \\
\hline Total & & & 6 & 11 & 22 & 6 & 4 & 6 & 55 \\
\hline
\end{tabular}

* Am: amarillo; A: azul; BC: blanco cristalino; BA: blanco ancho; C: crema; R: rojo. 


\section{Variables registradas}

Las características evaluadas fueron: días a floración femenina (DFF) y masculina (DFM), cuando el 50\% de las plantas de cada parcela presentaban emisión de estigmas o polen; acame de tallo (ACT) y de raíz (ACR) con base en el número de plantas que presentaron tallos quebrados o inclinación desde la base de la planta; y rendimiento $(\mathrm{RMz})$ con base en el peso total de mazorcas en cada parcela experimental. Todo esto con el objetivo de evaluar el comportamiento agronómico de las poblaciones. Al momento de la cosecha, la severidad de la pudrición (PuMz) por Fusarium spp., se determinó visualmente en cada mazorca de la unidad experimental, con base en la escala modificada de la usada por De León y Pandey (1989), donde: $1=0-20 ; 2=21-40 ; 3=41-60$; $4=61-80 ; 5=81-100 \%$, de granos con síntomas y se obtuvo la media ponderada en cada parcela con base en la ecuación siguiente:

Media ponderada de daño $=\left[\left(X_{1} \cdot Y_{1}\right)+\left(X_{2} \cdot Y_{2}\right)+\ldots\right.$ $\left.\left(\mathrm{X}_{5} \cdot \mathrm{Y}_{5}\right)\right] / \mathrm{T}$

donde: $\mathrm{X}_{\mathrm{i}}=$ número de mazorcas en cada categoría de la escala; $\mathrm{Y}_{\mathrm{i}}=$ valor de la escala; $\mathrm{T}=\left(\mathrm{X}_{1}+\mathrm{X}_{2}\right.$ $+\ldots+X_{5}$ ) número total de mazorcas en cada parcela experimental. Los valores más bajos se asumen como indicativos de menor susceptibilidad.

Los datos de severidad se transformaron por arco seno de la raíz cuadrada del daño porcentual, para tener una aproximación a la distribución normal (Steel y Torrie, 1980). Se identificaron las poblaciones de acuerdo con su procedencia geográfica y se obtuvo el ANDEVA y comparación de medias mediante la prueba de Tukey $(\mathrm{p} \leq 0,05)$ con el programa de análisis estadístico SAS System 9.0 (SAS, 2002); para cada ciclo de cultivo, se consideraron como fuentes de variación la procedencia y poblaciones anidadas en procedencia. La representación gráfica de las poblaciones de maíz se realizó con base en la agrupación por entidad de procedencia y color de grano. En cada caso, se calcularon promedios y desviaciones estándar. También se realizó un análisis de correlación entre rendimiento y severidad de la enfermedad.

\section{RESULTADOS Y DISCUSIÓN}

Se encontraron diferencias estadísticas significativas $(\mathrm{P} \leq 0,05)$ entre localidades, poblaciones, procedencias de las poblaciones e interacción localidad por procedencias en la evaluación de 2010 y 2011, tanto para pudrición de mazorca como en días a floración masculina y femenina y rendimiento por parcela. En el caso de la interacción localidades por poblaciones dentro de procedencias, no hubo diferencias significativas en pudrición de mazorca pero si en rendimiento y floración masculina $\mathrm{y}$ femenina (Cuadro 2). Los resultados muestran que las poblaciones, el origen y las localidades o agrosistemas de evaluación influyeron en la severidad de la pudrición de la mazorca.

Las diferencias significativas entre localidades y poblaciones son indicativos de las diferentes respuestas de las poblaciones al ambiente y muestran la diversidad fenotípica y genética en las variantes del maíz nativo del altiplano central mexicano. Esto se manifiestó por la significancia entre procedencias geográficas de las poblaciones de maíz, así como la significancia entre poblaciones de maíz dentro de procedencias geográficas (Cuadro 2).

\section{Efectos de localidad de evaluación}

La severidad de la enfermedad entre localidades fluctuó entre 28 y $55 \%$ en 2010 y entre 22 y $36 \%$ en 2011, por lo que son evidentes las diferencias en el grado de pudrición de mazorca en las poblaciones de maíz nativo, causada por el inóculo natural de Fusarium spp. Estos porcentajes fueron mayores a los encontrados por González et al. (2007) bajo condiciones de temporal en la región del Valle de Toluca $(6,1$ a 8,9\%). Las diferencias entre ambos resultados son consecuencia de los fenotipos evaluados, densidad natural del inóculo local y condiciones ambientales prevalecientes durante el desarrollo de cultivo en cada ciclo y en las regiones evaluadas. Las diferencias en la severidad puede atribuirse también a la variación diferencial en virulencia/agresividad entre aislamientos de Fusarium spp. de distintas localidades, tal como lo observaron Iglesias et al. (2010). 
Cuadro 2. Cuadrados medios de los análisis de varianza para pudrición de mazorca, y caracteres agronómicos en la evaluación de 55 poblaciones nativas de maíz de diversa procedencia geográfica, en Montecillo y Ayapango, Edo. de México y Huamelulpan, Oaxaca, México. 2010 y 2011.

\begin{tabular}{|c|c|c|c|c|c|}
\hline Fuentes de variación & GL & PuMz & PKg & DFM & DFF \\
\hline \multicolumn{6}{|l|}{ Ciclo 2010: } \\
\hline Localidades & 2 & $5682,0 * *$ & $137,7 * *$ & $10036,2 * *$ & $6702,5 * *$ \\
\hline Bloques/localidades & 6 & $75,0 \mathrm{NS}$ & $17,0 * *$ & $33,5 \mathrm{NS}$ & $43,8 \mathrm{NS}$ \\
\hline Procedencias & 5 & $960,5^{* *}$ & $3,7 * *$ & $1700,1 * *$ & $1349,6 * *$ \\
\hline Poblaciones (Procedencias) & 53 & $77,0^{*}$ & $1,1^{*}$ & $325,8^{* *}$ & $324,7 * *$ \\
\hline Localidad $\times$ Procedencias & 10 & $288,1 * *$ & $2,7 * *$ & $153,7 * *$ & $185,2 * *$ \\
\hline Localidades $\times$ Poblaciones/Procedencia & 101 & $65,0 \mathrm{NS}$ & $1,1^{*}$ & $23,2 * *$ & $29,4^{*}$ \\
\hline Error & 205 & 52,3 & 0,7 & 15,4 & 20,8 \\
\hline C.V. $(\%)$ & & 19 & 55 & 4 & 5 \\
\hline \multicolumn{6}{|l|}{ Ciclo 2011: } \\
\hline Localidades & 2 & $2040,1 * *$ & $6,1^{*}$ & $11789,5 * *$ & $11992,5 * *$ \\
\hline Bloques/localidades & 6 & $29,1 \mathrm{NS}$ & $3,1^{*}$ & $36,3 * *$ & $31,8^{*}$ \\
\hline Procedencias & 5 & $230,9^{* *}$ & $12,6^{* *}$ & $2513,0 * *$ & $2553,6^{* *}$ \\
\hline Poblaciones (Procedencias) & 53 & $42,9 * *$ & $2,6^{* *}$ & $530,5^{* *}$ & $559,2 * *$ \\
\hline Localidad $\times$ Procedencias & 10 & $96,3 * *$ & $20,0 * *$ & $59,7 * *$ & $81,4 * *$ \\
\hline Localidades $\times$ Poblaciones/Procedencia & 101 & $22,2 * *$ & $2,5 * *$ & $22,0 * *$ & $23,0^{* *}$ \\
\hline Error & 205 & 14,2 & 1,1 & 10,3 & 11,8 \\
\hline C.V. $(\%)$ & & 11 & 42 & 3 & 5 \\
\hline
\end{tabular}

NS: No significativo 5\%; *, ** Significativo con $\mathrm{P} \leq 0,05$ y $\mathrm{P} \leq 0,01$, respectivamente. GL: grados de libertad, PuMz: pudrición de mazorca, $\mathrm{PKg}$ : rendimiento de mazorca por parcela, DFM: días a floración masculina, DFF: días a floración femenina, C.V.: coeficiente de variación.

En 2010 la mayor severidad se presentó en Oaxaca $(53,6 \%)$, seguida de Montecillo $(35,8 \%)$ y Ayapango (27,5\%). En 2011, los valores en Montecillo $(35,4 \%)$ y Oaxaca $(34,0 \%)$ fueron estadísticamente equivalentes, pero superiores al de Ayapango $(22,2 \%)$ (Cuadro 3).

La menor severidad en la pudrición de mazorca por Fusarium spp. observada en Ayapango, puede atribuirse a las diferencias de oscilaciones térmicas entre localidades, ya que las temperaturas mínima y máxima promedio anual son más bajas $(5,5-21,6$ $\left.{ }^{\circ} \mathrm{C}\right)$ que en Huamelulpan $\left(10,5-24,4{ }^{\circ} \mathrm{C}\right)$ y Montecillo $\left(7,8-25{ }^{\circ} \mathrm{C}\right)(\mathrm{SMN}, 2000)$; además, la temperatura óptima de crecimiento de Fusarium spp. es de 22 a 28 ${ }^{\circ} \mathrm{C}$ (Carrillo, 2003). Esta última condición explicaría la mayor severidad observada en Montecillo, en ambos ciclos. En Huamelulpan-2010, los efectos de la enfermedad pudieron confundirse con los daños por la helada ocurrida en la etapa de formación de grano, que afectó severamente al cultivo, evento que muestra las diferencias en condiciones ambientales que pueden presentarse de un año a otro en una misma localidad.

\section{Efecto de poblaciones de maíz y procedencia geográfica}

Las poblaciones de maíz en cada localidad respondieron diferencialmente a Fusarium spp. bajo condiciones naturales, por lo que la interacción población x localidad en ambos ciclos fue significativa. En la Figura 1, se presenta la variación del daño entre poblaciones de maíz, la 53, 39, 52, 44 y 21 mostraron susceptibilidad, en contraste con la 35,31 , 2, 17 y 30 que se pueden considerar resistentes 
Cuadro 3. Porcentaje promedio de pudrición de mazorca (\%) y desviación estándar ( \pm ) de poblaciones de maíz agrupadas por procedencia y color de grano. Montecillo y Ayapango, Edo. de México y Huamelulpan, Oaxaca, México. 2010 y 2011.

\begin{tabular}{|c|c|c|c|c|c|c|c|c|}
\hline \multirow{2}{*}{$\begin{array}{l}\text { Grupos } \\
\text { poblacionales (n) }\end{array}$} & \multicolumn{3}{|c|}{ Ciclo 2010} & \multicolumn{3}{|c|}{ Ciclo 2011} & \multirow{2}{*}{$\begin{array}{c}\text { Prom. } \\
2010\end{array}$} & \multirow[t]{2}{*}{ Prom. 2011} \\
\hline & Mont-10 & Oax-10 & Aya-10 & Mont-11 & Oax-11 & Aya-11 & & \\
\hline \multicolumn{9}{|l|}{ Procedencia: } \\
\hline Edo. de Méx. (11) & $39,8 \pm 8,6$ & $60,0 \pm 19,5$ & $30,9 \pm 10,8$ & $39,5 \pm 11,9$ & $38,7 \pm 8,4$ & $24,0 \pm 2,5$ & $41,5 \pm 17,7$ & $34,1 \pm 11,1$ \\
\hline Guerrero (13) & $37,4 \pm 6,5$ & $55,5 \pm 17,5$ & $38,5 \pm 16,3$ & $37,3 \pm 10,5$ & $29,7 \pm 5,0$ & $23,1 \pm 2,6$ & $43,2 \pm 16,5$ & $30,0 \pm 9,0$ \\
\hline Oaxaca (13) & $27,4 \pm 4,4$ & $37,2 \pm 10,1$ & $25,8 \pm 5,3$ & $32,9 \pm 10,0$ & $26,0 \pm 3,8$ & $21,3 \pm 1,5$ & $29,6 \pm 8,4$ & $26,7 \pm 7,8$ \\
\hline Puebla (6) & $51,5 \pm 19,3$ & $58,3 \pm 21,5$ & $26,1 \pm 6,9$ & $37,7 \pm 6,8$ & $42,2 \pm 12,4$ & $22,4 \pm 2,5$ & $42,2 \pm 21,0$ & $32,9 \pm 11,7$ \\
\hline Tlaxcala (11) & $32,9 \pm 10,0$ & $50,6 \pm 15,0$ & $24,0 \pm 6,1$ & $32,3 \pm 6,8$ & $36,5 \pm 10,2$ & $21,9 \pm 2,1$ & $34,2 \pm 15,1$ & $30,3 \pm 9,4$ \\
\hline Testigo (1) & $25,6 \pm 3,3$ & $69,6 \pm 16,2$ & $20,0 \pm 1,9$ & $32,6 \pm 11$ & $30,9 \pm 7,2$ & $20,5 \pm 0,5$ & $35,8 \pm 24,2$ & $28,0 \pm 8,4$ \\
\hline Promedio (56) & 35,8 & 55,2 & 27,5 & 35,4 & 34,0 & 22,2 & 37,8 & 30,3 \\
\hline \multicolumn{9}{|l|}{ Color de grano: } \\
\hline Amarillo (6) & $38,1 \pm 20,2$ & $46,0 \pm 11,3$ & $24,3 \pm 8,9$ & $30,8 \pm 8,0$ & $31,7 \pm 11,4$ & $22,0 \pm 2,2$ & $34,8 \pm 16,4$ & $27,9 \pm 9,0$ \\
\hline Azul (12) & $34,9 \pm 10,4$ & $49,2 \pm 20,6$ & $27,7 \pm 9,0$ & $38,8 \pm 12,2$ & $31,8 \pm 7,9$ & $22,5 \pm 2,8$ & $36,0 \pm 16,2$ & $31,0 \pm 10,8$ \\
\hline Blanco C (22) & $37,1 \pm 11,6$ & $49,9 \pm 18,1$ & $27,9 \pm 8,1$ & $33,9 \pm 9,2$ & $34,7 \pm 10,2$ & $22,2 \pm 2,2$ & $36,0 \pm 15,4$ & $30,2 \pm 9,9$ \\
\hline Blanco A (6) & $36,6 \pm 6,7$ & $63,2 \pm 13,0$ & $43,3 \pm 18,5$ & $37,8 \pm 8,5$ & $32,6 \pm 5,9$ & $23,6 \pm 2,5$ & $47,2 \pm 17,7$ & $31,3 \pm 8,4$ \\
\hline Crema (4) & $39,8 \pm 10,0$ & $68,9 \pm 18,9$ & $29,3 \pm 14,5$ & $39,5 \pm 4,4$ & $42,9 \pm 9,4$ & $23,6 \pm 2,5$ & $42,4 \pm 21,4$ & $35,3 \pm 10,4$ \\
\hline Rojo (6) & $29,9 \pm 6,0$ & $54,8 \pm 15,2$ & $29,3 \pm 11,1$ & $33,9 \pm 11,1$ & $26,7 \pm 2,8$ & $22,4 \pm 2,2$ & $34,3 \pm 13,4$ & $27,7 \pm 8,2$ \\
\hline
\end{tabular}

n= número de poblaciones de maíz en cada grupo; número de observaciones en cada localidad en $2010=\mathrm{n} \times 2 ; 2011=\mathrm{n} \times 3$; total de observaciones $2010=\mathrm{n} \times 6$; 2011= $\mathrm{n} \times 9$.

Mont: Montecillo; Oax: Huamelulpan; Aya: Ayapango.

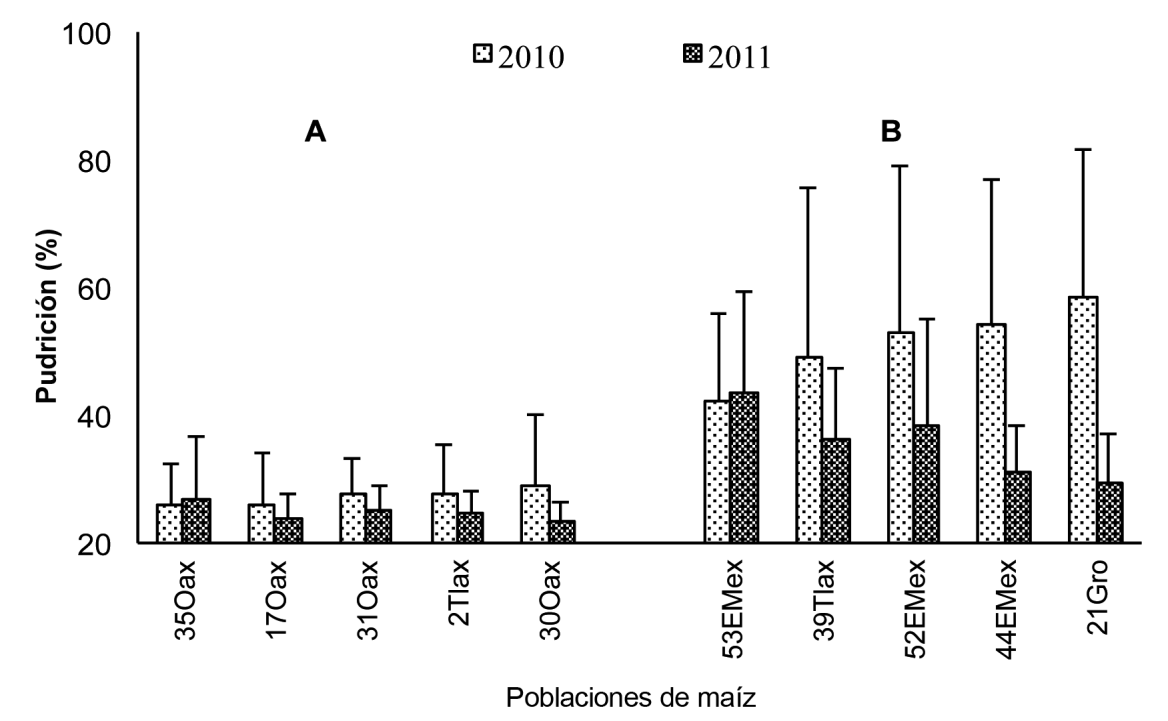

Figura 1. Promedios de pudrición de mazorca de poblaciones nativas de maíz representativas de resistencia (A) y susceptibilidad (B), en tres localidades: Montecillo y Ayapango, Edo. de México y Huamelulpan, Oaxaca. 2010 y 2011. 
o menos susceptibles; el resto de poblaciones se encontraron entre los valores de 20 y $60 \%$ de daño. Sin embargo, para confirmar la resistencia o tolerancia a la enfermedad observada en campo, es necesaria la evaluación con inoculación artificial para descartar el escape a la enfermedad.

Las poblaciones procedentes de los estados de México y Puebla mostraron en promedio mayor porcentaje de daño, mientras que las de Oaxaca presentaron menor daño en todos los ambientes (Cuadro 3, Figura 2). El peso de mazorca por parcela (rendimiento) fue disminuido tanto por la severidad de la enfermedad, como por los daños por helada, fenómeno que fue más notorio en Oaxaca-2010. No obstante, en ambos ciclos de cultivo las poblaciones agrupadas por procedencia mostraron rendimientos diferenciales.

En la localidad de Montecillo, destacaron con mayor rendimiento las procedentes de Oaxaca y el testigo, mientras que en Oaxaca las de mayor rendimiento fueron las poblaciones locales y las de Guerrero, y en Ayapango fueron las procedentes de Oaxaca, Estado de México y Tlaxcala (Figura 3).

La relación entre rendimiento y severidad de la enfermedad fue inversa $(\mathrm{r}=-0,35493 ; \mathrm{p}=<0,0001$ $\mathrm{n}=373$ en 2010 y $\mathrm{r}=-0,3339 ; \mathrm{p}=<0,0001 \mathrm{n}=501$ en 2011). Las poblaciones 52 y 16 muestran como la severidad de la enfermedad afectó al rendimiento, contrario a la 10 y 26 con menor daño y mayor peso de mazorca por parcela; mientras que las poblaciones 50,2 y 1 pueden ser tolerantes al obtener rendimientos aceptables a pesar del daño (Figura 4).

\section{Interacción procedencia geográfica por localidad}

Los valores de la interacción procedencia por localidad (Cuadros 4 y 5), fueron diferentes tanto en magnitud como en sentido, lo cual evidencia claramente la respuesta diferencial de los genotipos al ambiente. Así mismo, se relaciona con la naturaleza poligénica de la resistencia a la pudrición de mazorca por Fusarium spp. en maíz (Ali et al., 2005). La respuesta diferencial percibida en los agrupamientos por procedencia geográfica, puede relacionarse con la capacidad de adaptación propia de los maíces nativos, ya sea a un determinado ambiente o a varios, como lo mostraron las poblaciones provenientes de Oaxaca, que prosperaron bien en los tres ambientes de evaluación.

Ehrlich y Raven (1964) mencionan que entre poblaciones de organismos estrechamente relacionados cada una ejerce una fuerza selectiva sobre la otra, originando respuestas adaptativas recíprocas como consecuencia de la interacción ecológica conocida como coevolución, la cual, podría impulsar la diversificación de la vida de

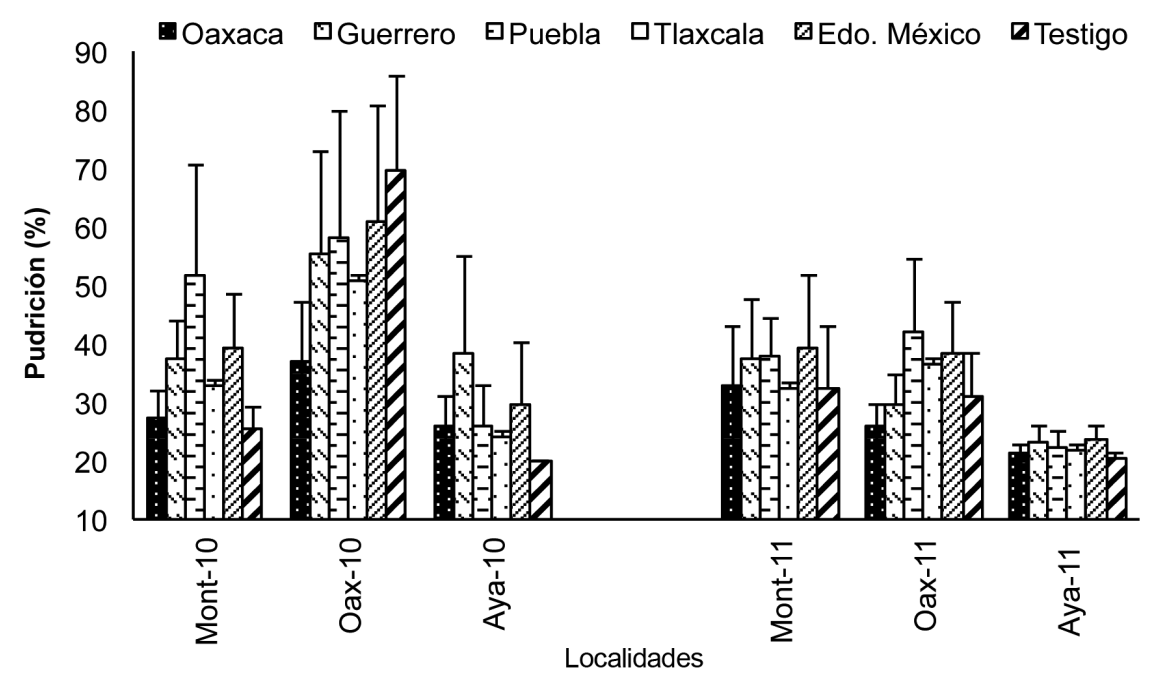

Figura 2. Pudrición de mazorca (\%) de poblaciones de maíz agrupadas por procedencia geográfica, evaluadas en tres localidades: Montecillo y Ayapango, Edo. de México y Huamelulpan, Oaxaca. 2010 y 2011. 


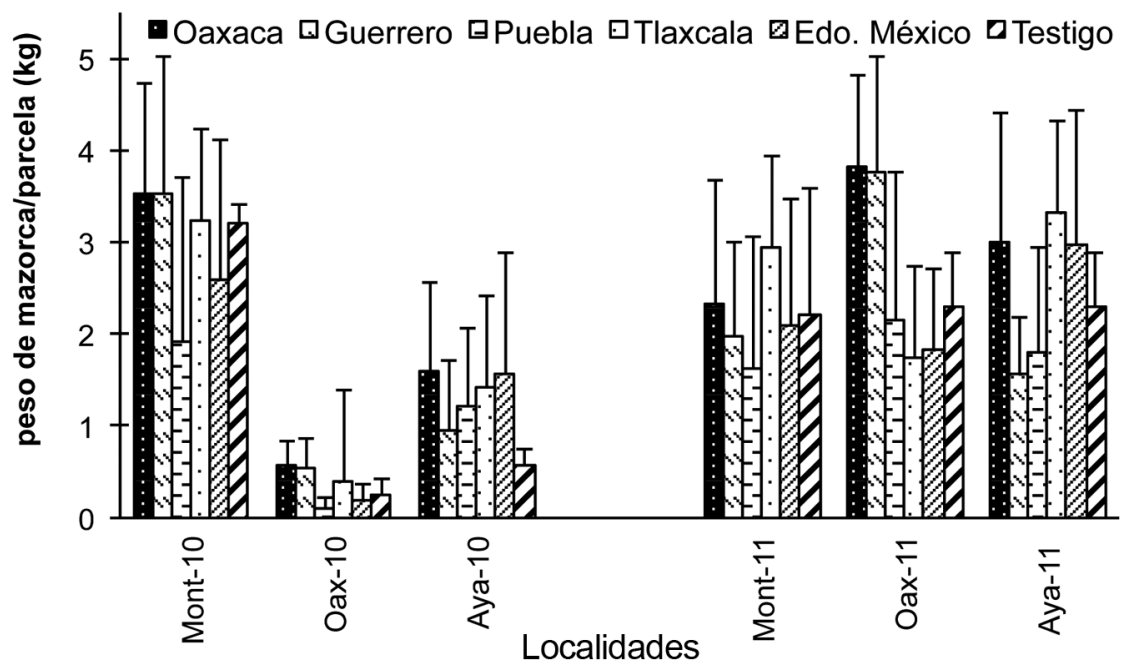

Figura 3. Rendimiento de mazorca por parcela de poblaciones de maíz, agrupadas por procedencia geográfica, evaluadas en tres localidades: Montecillo y Ayapango, Edo. de México y Huamelulpan, Oaxaca. 2010 y 2011.

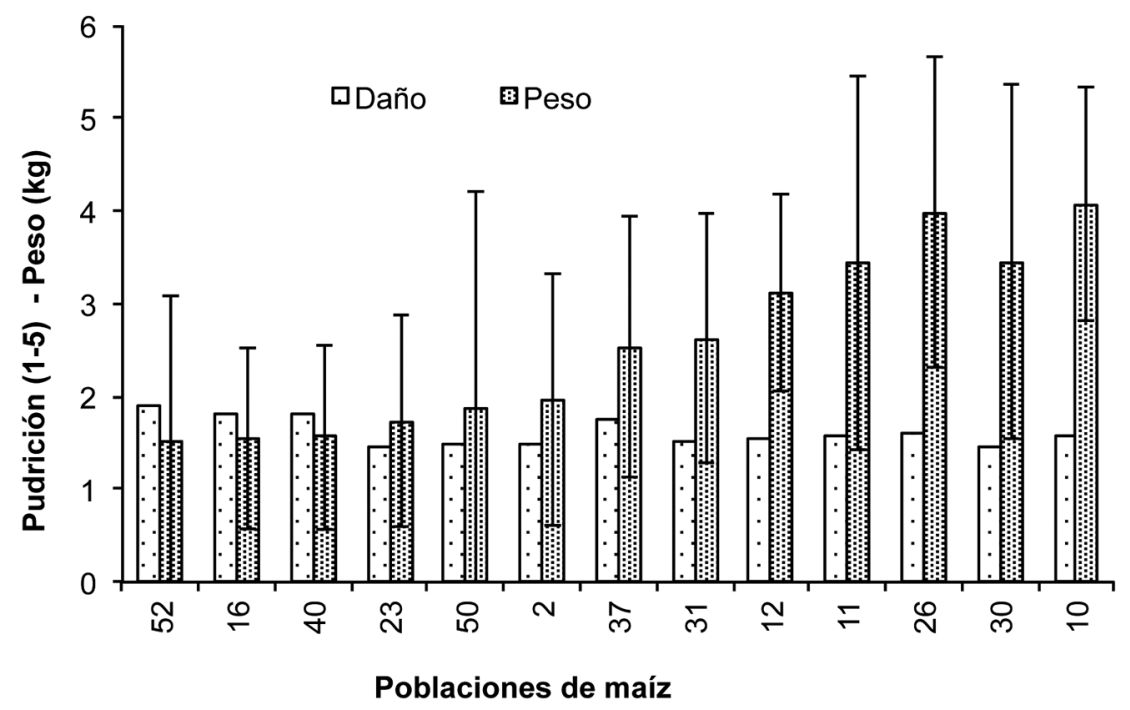

Figura 4. Genotipos representativos del daño promedio por pudrición en el rendimiento de mazorca, en tres localidades de evaluación: Montecillo y Ayapango, Edo. de México y Huamelulpan, Oaxaca. 2011.

forma sistemática. Lo anterior hace suponer que las poblaciones de maíz nativo y las especies de Fusarium de una región-ambiente determinado coevolucionan, y han logrado coadaptarse, pues en este estudio se observó cómo las poblaciones de origen cercano al lugar de evaluación, presentaron menor severidad y su rendimiento fue mejor.

Por otro lado, al introducir poblaciones de otras condiciones ecológicas, estas pueden presentar mayor susceptibilidad a las poblaciones de patógenos locales, un 
Cuadro 4. Efectos de interacción de procedencia geográfica x localidad, para pudrición de mazorca (\%), Montecillo y Ayapango, Edo. de México y Huamelulpan, Oaxaca. 2010 y 2011.

\begin{tabular}{lcccccccc}
\hline & Promedio & \multicolumn{3}{c}{ Interacción } & \multicolumn{3}{c}{ Promedio } & \multicolumn{3}{c}{ Interacción } \\
\hline Origen (n) & $\mathbf{2 0 1 0}$ & Mont-10 & Oax-10 & Aya-10 & $\mathbf{2 0 1 1}$ & Mont-11 & Oax-11 & Aya-11 \\
\hline Puebla (6) & $\mathbf{4 5 , 3}$ & 9,903 & $-2,730$ & $-7,280$ & $\mathbf{3 4 , 1}$ & $-1,283$ & 4,600 & $-3,400$ \\
Edo. de Méx. (12) & $\mathbf{4 3 , 6}$ & 0,173 & 0,940 & $-0,510$ & $\mathbf{3 4 , 1}$ & 0,717 & 1,300 & $-1,600$ \\
Guerrero (13) & $\mathbf{4 3 , 8}$ & $-2,697$ & $-4,030$ & 6,620 & $\mathbf{3 0 , 0}$ & 2,387 & $-3,830$ & 1,370 \\
Tlaxcala (11) & $\mathbf{3 5 , 8}$ & 0,773 & $-0,960$ & 0,090 & $\mathbf{3 0 , 2}$ & $-2,813$ & 2,770 & $-0,030$ \\
Testigo (1) & $\mathbf{3 8 , 4}$ & $-9,097$ & 15,470 & $-6,480$ & $\mathbf{2 8 , 0}$ & $-0,283$ & $-0,600$ & 0,800 \\
Oaxaca (13) & $\mathbf{3 0 , 1}$ & 0,973 & $-8,660$ & 7,590 & $\mathbf{2 6 , 7}$ & 1,287 & $-4,230$ & 2,870 \\
Promedio (56) & 39,5 & & & & 30,5 & & & \\
\hline
\end{tabular}

Mont: Montecillo; Oax: Huamelulpan; Aya: Ayapango.

Cuadro 5. Efectos de interacción de procedencia geográfica x localidad, para rendimiento de mazorca por parcela (kg). Montecillo y Ayapango, Edo. de México y Huamelulpan. Oaxaca. 2010 y 2011.

\begin{tabular}{lcccccccc}
\hline & Promedio & \multicolumn{3}{c}{ Interacción } & \multicolumn{3}{c}{ Promedio } & \multicolumn{3}{c}{ Interacción } \\
\cline { 2 - 9 } Origen (n) & $\mathbf{2 0 1 0}$ & Mont-10 & Oax-10 & Aya-10 & $\mathbf{2 0 1 1}$ & Mont-11 & Oax-11 & Aya-11 \\
\hline Edo. de Méx. (12) & $\mathbf{1 , 5}$ & $-0,272$ & $-0,039$ & 0,478 & $\mathbf{2 , 3}$ & 0,105 & $-0,592$ & 0,533 \\
Guerrero (13) & $\mathbf{1 , 7}$ & 0,328 & 0,061 & $-0,422$ & $\mathbf{2 , 5}$ & $-0,181$ & 1,178 & $-1,027$ \\
Oaxaca (13) & $\mathbf{1 , 9}$ & 0,128 & $-0,139$ & $-0,022$ & $\mathbf{3 , 0}$ & $-0,381$ & 0,678 & $-0,127$ \\
Puebla (6) & $\mathbf{1 , 1}$ & $-0,639$ & 0,194 & 0,411 & $\mathbf{1 , 9}$ & 0,019 & 0,178 & $-0,227$ \\
Tlaxcala (12) & $\mathbf{1 , 7}$ & 0,061 & $-0,106$ & 0,011 & $\mathbf{2 , 7}$ & 0,519 & $-1,022$ & 0,473 \\
Testigo (1) & $\mathbf{1 , 3}$ & 0,395 & 0,028 & $-0,455$ & $\mathbf{2 , 5}$ & 0,019 & $-0,322$ & 0,473 \\
Promedio (56) & 1,5 & & & & 2,5 & & & \\
\hline
\end{tabular}

Mont: Montecillo; Oax: Huamelulpan; Aya: Ayapango.

ejemplo de esta situación se observó con las poblaciones procedentes de la región Chalco-Amecameca en ambos ciclos de evaluación y en las dos localidades distantes a su origen; al respecto, Ortega (2003) ya había observado este fenómeno con poblaciones de esa misma región llevadas a Montecillo, aún cuando ambas regiones son similares en ubicación y clima. Otro ejemplo fue la susceptibilidad al carbón de la espiga (Sporisorium reilianum) del híbrido H-353 liberado para el Bajío con $50 \%$ de germoplasma tropical.

La respuesta de las poblaciones de maíz agrupadas por su origen geográfico, al inoculo de Fusarium spp. presente en las localidades de evaluación fue diferencial, ya que los maíces locales tienden a presentar menor pudrición de mazorca y mayor rendimiento, efecto que ya ha sido observado en estudios como los de Muñoz et al. (2001), Ramírez et al. (2003) y Alvarado (2010), los cuales muestran la existencia de una amplia diversidad entre materiales nativos, concluyendo que están en constante evolución, con una evidente adaptación específica al medio ambiente que los rodea, gracias a la selección hecha por los campesinos de la región.

La tendencia observada en los efectos de interacción respaldan la propuesta de resolver los problemas del cultivo con un enfoque endógeno con 
investigación y mejoramiento participativo a partir del patrimonio genético local del maíz. La base de partida del proceso de mejoramiento en términos de sanidad de la mazorca representa una ventaja inicial, acompañada de otras respuestas de coadaptación y productividad. Además, de esta manera se promovería la continuidad de la evolución bajo domesticación, sujeta a los factores selectivos del medio específico que difiere entre microrregiones ecológicas.

\section{Influencia de color de grano}

Los materiales de endospermo amarillo o pericarpio rojo presentaron menor pudrición de mazorca de manera consistente en todas las localidades (Figura 5), seguidos de los de color azul, mientras que los de color blanco y crema, fueron los más susceptibles.

Se sabe que el grano de maíz con pigmento tipo flavonoides (rojo, azul, morado y negro) debe su coloración a las antocianinas, el grupo más importante de pigmentos vegetales solubles al agua y visibles (López-Martínez et al., 2009), mientras que en el maíz amarillo son los carotenoides, solubles en grasa (Farré et al., 2010). En este trabajo los resultados no muestran una relación directa entre coloraciones de grano con porcentajes de pudriciones de la mazorca y en cierta forma, las evaluaciones de campo, indicaron independencia del ataque de Fusarium y coloraciones de granos en las poblaciones nativas de maíz (Figura 5). En otros trabajos se ha encontrado una relación directa con la textura de grano, tal como lo señalan Antonio et al. (2004) en el caso de la susceptibilidad a las pudriciones de mazorca en las variedades de maíz de pericarpio/aleurona azul.

Con relación a rendimiento base por grupos de coloraciones de grano, en condiciones de estaciones y ambientesfavorables(Montecillo-2010y Oaxaca-2011), las poblaciones de grano blanco y rojo presentaron un mayor rendimiento por parcela experimental. Por otro lado, cuando las condiciones son desfavorables (Oaxaca-2010) los grupos de poblaciones no difieren en rendimiento. Esto es indicativo de la interacción de las poblaciones con el ambiente (Figura 6).

Respecto al acame, González et al. (2007) encontraron que el incremento en la pudrición de mazorca está asociado con el aumento en el acame, y que ambas características afectan al rendimiento. Aunque en este estudio el acame no fue significativo, en el mejoramiento de poblaciones de maíz nativos no se debe olvidar la resistencia al acame y de esta manera contribuir indirectamente a resolver el problema de pudrición de mazorca.

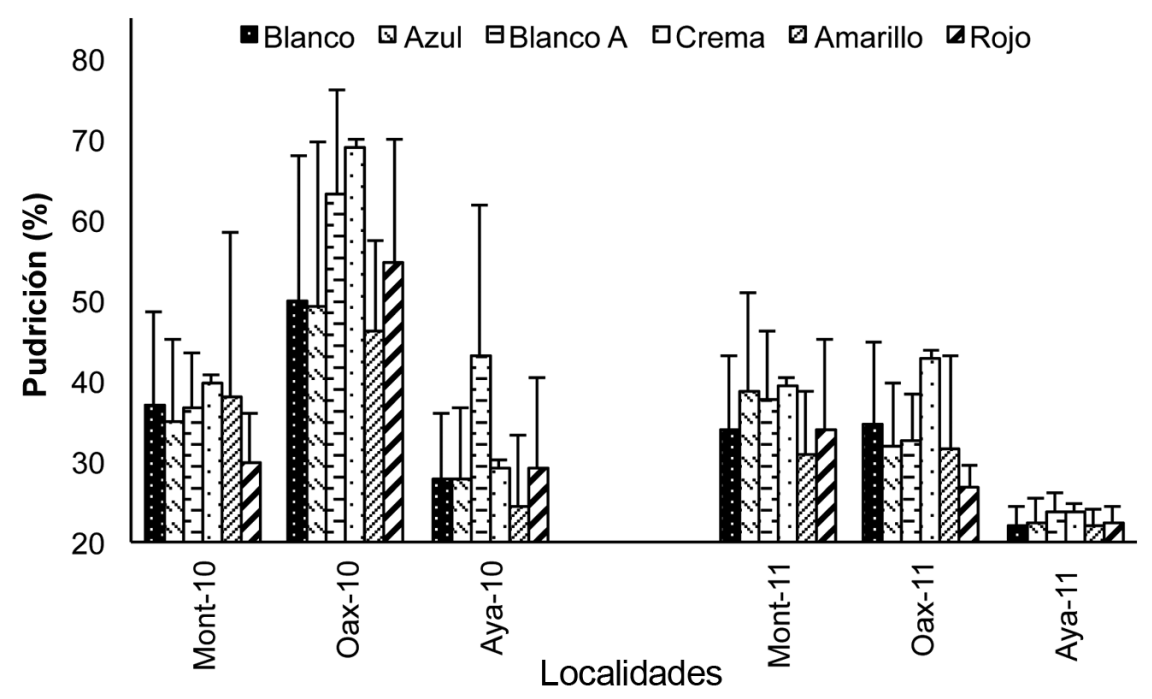

Figura 5. Pudrición de mazorca (\%) por Fusarium spp. de 56 poblaciones de maíz, agrupadas por su color de grano, en tres localidades de evaluación: Montecillo y Ayapango, Edo. de México. y Huamelulpan, Oaxaca. 2010 y 2011. 


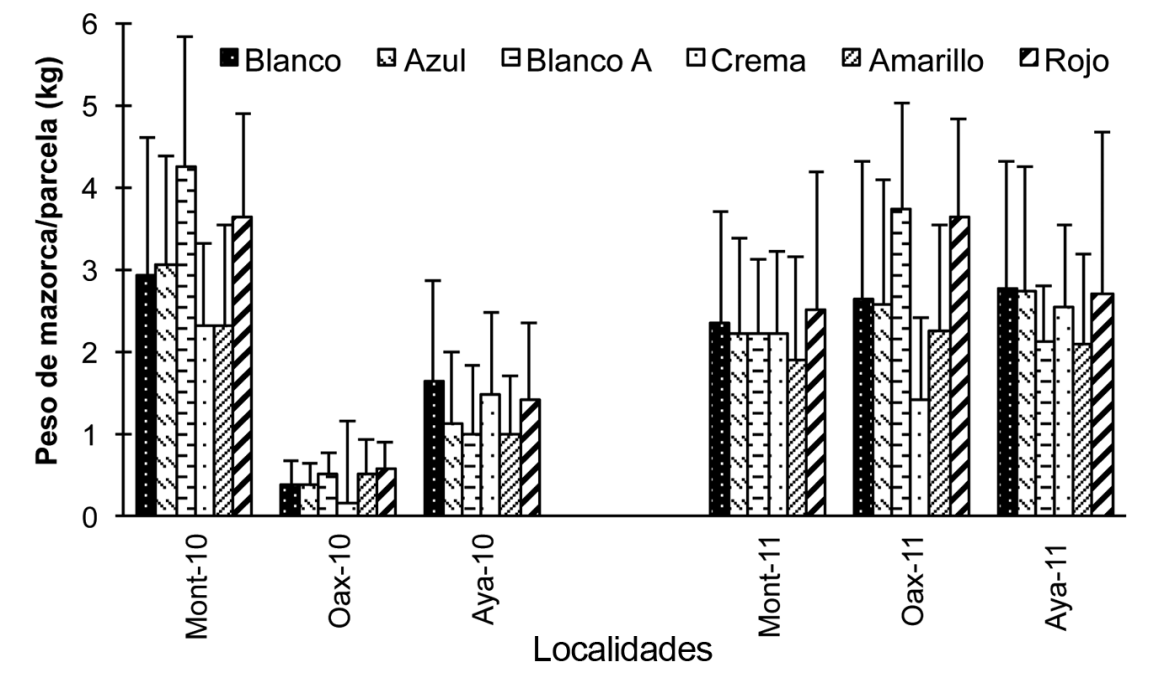

Figura 6. Rendimiento promedio de poblaciones de maíz (kg), agrupadas por color de grano, en tres localidades de evaluación: Montecillo y Ayapango, Edo. de México y Huamelulpan, Oaxaca. 2010 y 2011.

La presencia de pudriciones de mazorca causada por Fusarium spp., en las tres localidades de evaluación de la región templada y central de México, muestran tres aspectos relevantes: la prevalencia del patógeno en las parcelas de cultivo y severidad de la enfermedad, la respuesta diferencial de las poblaciones agrupadas por origen geográfico, muestran diferentes grados de susceptibilidad y mecanismo asociados a la tolerancia o resistencia al patógeno; y los resultados muestran interacciones patógeno-hospedero del tipo Fusarium spp. - poblaciones de maíz. Todo esto bajo la presencia de inóculo natural. Oren et al. (2003), Presello (2004), Dejardins et al. (2005), Dejardins et al. (2007) y Löffler et al. (2010) han demostrado que las especies de Fusarium interactúan con las distintas poblaciones de maíz que son sembrados (coevolución), y es posible encontrar fuentes de resistencia en las poblaciones aun cuando dependen de las condiciones ambientales para favorecer la presencia de Fusarium.

Dado que las poblaciones no han sido sometidas a procesos controlados de mejoramiento, los resultados muestran el alto potencial de las poblaciones nativas para su utilización como fuentes de resistencia a Fusarium spp. y de rendimiento, en programas de mejoramiento genético convencionales y moleculares.

Es necesario estudiar con mayor detalle y profundidad los patosistemas para entender la relación planta-patógeno en términos de diversidad genética y mecanismos de coevolución y coadaptación entre especies, y poder dar solución al problema de las enfermedades en cultivos de interés agronómico.

\section{AGRADECIMIENTOS}

Al Dr. Porfirio Ramírez Vallejo ${ }^{\dagger}$ (Q. E. P. D.) por sus enseñanzas y aportes a esta investigación.

\section{LITERATURA CITADA}

Ali, M.L., J.H. Taylor, L. Jie, G. Sun, M. William, K.J. Kash, L.M. Reid, y K.P. Pauls. 2005. Molecular mapping of QTLs for resistance to Gibberella ear rot, in corn, caused by Fusarium graminearum. Genome 48:521-533.

Alvarado, B.G. 2010. Diversidad de maíces nativos de tres nichos ecológicos del Altiplano Poblano-Tlaxcalteca. Tesis doctorado, Colegio de Postgraduados en Ciencias Agrícolas, Montecillo, Estado de México.

Antonio, M.M., J.L. Arellano, S.G. García, C.S. Miranda, J.A. Mejía, y F.V. González. 2004. Variedades criollas de maíz azul raza chalqueño. Características agronómicas y calidad de semilla. Rev. Fitotec. Mex. 27:9-15. 
Boege, E. 2010. El patrimonio biocultural de los pueblos indígenas de México. Hacia la conservación in situ de la biodiversidad y agrobiodiversidad en los territorios indígenas. Instituto Nacional de Antropología e Historia. México, D.F. 342 p.

Carrillo, L. 2003. Los hongos de los alimentos y forrajes. Univ. Nal. de Salta. Rep. Argentina.

Castillo, F., E. Herrera, J. Romero, R. Ortega, M. Goodman, y M. Smith. 2000. Diversidad genética del maíz y su aprovechamiento in situ a nivel regional. En: CIAT, editor, Fitomejoramiento Participativo en América Latina y el Caribe. Mem. Simp. Internacional. Quito, Ecuador. Ago 31-Sep. 3. 1999. CIAT - Programa PRGA del CGIAR. Cali, Colombia. 7 p.

De León, C., y S. Pandey. 1989. Improvement of resistance to ear and stalk rots and agronomic traits in tropical maize gene pools. Crop Sci. 29:12-17.

Desjardins, A.E., R.D. Plattner, y P.E. Nelson. 1994. Fumonisin production and other traits of Fusarium moniliforme strains from maize in northeast Mexico. Appl. Environ. Microbiol. 60:1695-1697.

Desjardins, A.E., R.D. Plattner, R.J. Stessman, S.P. McCormick, y M.J. Millard. 2005. Identification and heritability of fumonisin insensitivity in Zea mays. Phytochemistry 66:2474-2480.

Desjardins, A.E., C.M. Maragos, y R.H. Proctor. 2006. Maize ear rot and moniliformin contamination by cryptic species of Fusarium subglutinans. J. Agric. Food Chem. 54:7383-90.

Desjardins, A.E., M. Busman, M. Muhitch, y R.H. Proctor. 2007. Complementary host-pathogen genetic analyses of the role of fumonisins in the Zea mays-Gibberella moniliformis interaction. Physiol. Mol. Plant Pathol. 70:149-160.

Ehrlich, P.R., y P.H. Raven. 1964. Butterflies and plants: a study in coevolution. Evolution. 18:586-608.

Farhang, A., M. Mansooreh, E. Masoud, F.M. Seyedeh, y R. Karami-O. 2007. Natural occurrence of Fusarium species in maize kernels at Gholestan provience in northern Iran. Asian J. Plant Sci. 6:1276-1281.

Farré, G., G. Sanahuja, S. Naqvi, C. Bai, T. Capell, C. Zhu, y P. Christou. 2010. Travel advice on the road to carotenoids in plants. Plant Sci. 179:28-48.

Gallardo, R.E., M. Ibarra, M. Sánchez, C. Cuamea, G. Molina, V. Parra, B. Rosas, y R. Cortez. 2006. Micobiota de maíz (Zea mays L.) recién cosechado y producción de fumonisina BI por cepas de Fusarium verticillioides (Sacc.) Nirenb. Rev. Mex. Fitopat. 24:27-34.
García, E. 1973. Modificaciones al sistema de clasificación climática de Köppen. 2 ed. Inst. de Geografía, UNAM, México.

García-Aguirre, G., y R. Martínez-Flores. 2010. Especies de Fusarium en granos de maíz recién cosechado y desgranado en el campo en la región de Ciudad Serdán, Puebla. Revista Mexicana de Biodiversidad 81:15-20.

Gimeno, A., y M.L. Martins. 2011. Micotoxinas y micotoxicosis en animales y humanos. 3 ed. Special nutients Inc. Miami, FL, USA.

González, H., G. Vázquez, C. Sahagún, P. Rodríguez, y L. Pérez. 2007. Rendimiento del maíz de temporal y su relación con la pudrición de mazorca. Agric. Téc. México 33:33-42.

Hernández, X.E. 1985. Biología agrícola. Los conocimientos biológicos y su aplicación a la agricultura. CECSA. México DF.

Iglesias, J., D. Presello, G. Botta, G. Lori, y C. Fauguel. 2010. Aggressiveness of Fusarium Section Liseola isolates causing maize ear rot in Argentina. J. Plant Pathol. 92(1):205-211.

INAFED (Instituto Nacional para el Federalismo y el Desarrollo Municipal). 2014. Enciclopedia de los Municipios y Delegaciones de México. http://www.elocal.gob.mx/wb/ELOCAL/ELOC_Enciclopedia (Consultado 18 julio 2014).

Löffler, M., B. Kessel, M. Ouzunova, y T. Miedaner. 2010. Population parameters for resistance to Fusarium graminearum and Fusarium verticillioides ear rot among large sets of early, mid-late and late maturing European maize (Zea mays L.) inbred lines. Theor. Appl. Genet. 120:1053-62.

López-Martínez, L.X., R.M. Oliart-Ros, G. Valerio-Alfaro, C-H Lee, K.L. Parkin, y H.S. Garcia. 2009. Antioxidant activity, phenolic compounds and anthocyanins content of eighteen strains of Mexican maize. LWTFood Sci. Technol. 42:1187-1192.

Mesterházy, A., M. Lemmens, y L.M. Reid. 2012. Breeding for resistance to ear rots caused by Fusarium spp. in maize - a review. Plant Breeding 132:1-19.

Morales-Rodríguez, I., M.J. Yáñez-Morales, H.V. SilvaRojas, G. García-de-los-Santos, y D.A. Guzmánde-Peña. 2007. Biodiversity of Fusarium species in Mexico with ear rot in maize, and their identification using a phylogenetic approach. Mycopathologia 163:31-39.

Muñoz, O.A., A. Santacruz, J.I. Olvera, O. Taboada, y J.A. Cuevas. 2001. Diversidad del maíz en los nichos 
ecológicos y culturales de México. Universidad Autónoma Chapingo, Programa Nal. Etnobotánica. Serie Agroecosistemas. $N^{\circ}$ 5, 31 p. México, http:// www.chapingo.mx/bagebage/18.pdf (Consultado 18 jul. 2014).

Nankam, C., y J.K. Pataky. 1996. Resistance to kernel infection by Fusarium moniliforme in the sweet corn inbred IL125b. Plant Dis. 80:593-598.

Oren, L., S. Ezrati, D. Cohen, y A. Sharon. 2003. Early events in the Fusarium verticillioides-maize interaction characterized by using a green fluorescent protein-expressing transgenic isolate. Appl. Environ. Microbiol. 69:1695-1701.

Ortega, R. 2003. La diversidad del maíz en México. En: G. Esteva, y C. Marielle, Coord., Sin maíz no hay país. Culturas Populares, CONACULTA, Museo Nal. de Culturas Populares. México, D. F. p. 123-154.

Palau, M.T. 2000. Relación hospedero-parásito Trypanosoma cruzi. MVZ-Córdoba 5:33-37.

Presello, D.A., L.M. Reid, y D.E. Mather. 2004. Resistance of argentine maize germplasm to Gibberella and Fusarium ear rots. Maydica 49:73-81.

Presello, D., J. Iglesias, G. Botta, G.T. Eyherabide. 2007. Severity of Fusarium ear rot and concentration of fumonisins in Argentinian maize hybrids. Crop Protec. 26:852-855.

Ramírez, A.M., M. Salinas, y O. Taboada. 2003. Maíz azul de los Valles Altos de México. I. Rendimiento de grano y caracteres agronómicos. Rev. Fitotec. Mex. 26:101-107.

Robledo, M.L., S. Marín, y A.J. Ramos. 2001. Contaminación natural con micotoxinas en maíz forrajero y granos de café verde en el Estado de Nayarit (México). Rev. Iberoam. de Micol. 18:141-144.

Rocha, G., Z. Lozano, y L. Martínez. 2004. Mecanismos de patogenicidad e interacción: parásito-Hospedero II. BUAP. Puebla, México.

SAS Institute. 2002. Version 9.0. SAS Inst. Cary. NC. USA.

SIAP (Servicio de Información Agroalimentaria y Pesquera). 2013. Estadísticas de siembras y cosechas. http:// www.siap.gob.mx (Consultado 9 octubre 2014).

Smith, M., F. Castillo, y F. Gómez. 2001. Participatory plant breeding with maize in Mexico and Honduras. Euphytica 122:551-565.

SMN (Servicio Meteorológico Nacional). 2000. Normales climatológicas. http://smn.cna.gob.mx (Consultado 30 junio 2013).

Steel, R.G., y J.H. Torrie. 1980. Principle and procedures of statistics. A biometrical approach. McGrow Hill Kogakusha, Ltd. Tokyo, Japan.

Thomas, M.D., y I.W. Buddenhagen. 1980. Incidence and persistence of fusarium moniliforme in symptomless maize kernels and seedlings in Nigeria. Mycologia 72:882-887.

Trung, T.S., C. Tabuc, S. Bailly, A. Querin, P. Guerre, y J.D. Bailly. 2008. Fungal mycoflora and contamination of maize from Vietnam with aflatoxin B1 and fumonisin B1. World Mycotoxin Journal 1(1):87-94.

Vera-Guzmán, A.M., J.L. Chávez-Servia, J.C. CarrilloRodríguez. 2012. Proteína, lisina y triptófano en poblaciones nativas de maíz Mixteco. Rev. Fitotec. Mex. 35:7-13. 
